\title{
Effective Masses for Donor Binding Energies in Non-Magnetic and Magnetic Quantum Well Systems: Effect of Magnetic Field
}

\author{
S. Rajashabala and K. Navaneethakrishnan \\ School of Physics, Madurai Kamaraj University, Madurai-625021, India
}

Received on 24 May, 2007

\begin{abstract}
Donor binding energies in quantum well systems using position dependent effective masses are obtained. Results are provided for $\mathrm{GaAs}-\mathrm{Ga}_{1-x} \mathrm{Al}_{x} \mathrm{As}$ and the semimagnetic $\mathrm{CdTe}-\mathrm{Cd}_{1-x} \mathrm{Mn}_{x} \mathrm{Te}$ systems. In the latter the barrier height reduces in a magnetic field. Using the available experimental data the variations of the barrier height with magnetic field has been obtained using a simple model. Our results are in good agreement with similar works in the literature in the case of constant effective mass for the donor electron. However, in the case of the semi magnetic system and in the case of the position dependent effective mass, the results obtained are shown to be appreciably different for narrow well dimensions. The validity of the effective mass approximation in the case of applied magnetic field is also critically examined.
\end{abstract}

Keywords: Quantum wells

\section{INTRODUCTION}

The effective mass theory (EMT) [1] enunciated by Kohn and Luttinger is very useful in the study of impurity states in semiconductors. Apart from many valley semi conductors $[2,3]$ such as $\mathrm{Si}, \mathrm{Ge}, \mathrm{GaP}$ etc. in almost all situations this theory is used in interpreting the rich electrical and optical data. This theory in its simplest form assumes a hydrogen like donor atom immersed in a dielectric background. The energy eigenvalues of such a system can be obtained using a potential $\boldsymbol{V}(\boldsymbol{r})=e / \varepsilon(r) r$ where $\varepsilon(r)$ is the static dielectric screening function and $r$ is the distance between the donor ion and electron. Since the static dielectric constant $\varepsilon$ and the effective mass $m^{*}$ at the bottom of conduction band are around 10 and $0.1 m_{0}$ respectively ( $m_{0}$ is free electron mass) for a typical semiconductor, the impurity orbits are quite large, $\sim 100 \AA$. Hence the screening function may be replaced by a static dielectric constant in the potential energy term of the Hamiltonian. This theory rests on two basic approximations [1]:

1) $E_{\text {ion }} a / \Delta E_{a}^{*}<<1$ where $\mathrm{E}_{\mathrm{ion}}$ is donor ionization energy, $\Delta E$ is the average separation between two bands, $a$ the lattice constant, $a^{*}$ the effective Bohr radius defined as $a^{*}=\varepsilon \hbar^{2} / m^{*} e^{2}$, and

$2)|\kappa| / \kappa_{B Z}=a / 6.2 a^{*}<<1$ where $|\kappa|$ is approximately equal to $1 / a^{*}$ and $\kappa_{B Z}$ is the wave vector of the radius of the Brillouin zone approximating the real Brillouin zone of the system, which is $\sqrt[3]{24 \pi^{2} / a^{3}}=6.2 / a$

Low dimensional semiconductor systems have attracted considerable attention in the last two decades. After the pathbreaking work of Bastard [4], several researchers have contributed to the understanding of the optical and electronic properties of impurities in quantum well (QW) systems. This includes quantum well wires, quantum dots and superlattices. Several novel superlattice systems have been fabricated and rich interesting physics has come out of such studies $[5,6]$. In a QW system when the well dimension is much smaller than the donor effective Bohr radius, the EMT requires close scrutiny, since the potential varies rapidly at the barriers. In almost all studies so far the EMT has been used even for well dimensions of the order of a mono layer or so $(<10 \AA)$. Interestingly, the quantum effects such as the quantum size effect manifest under strong confinement, which requires well dimensions ranging from $10 \AA$ to about $200 \AA$ or so. Hence applications of the EMT to such confined systems require a critical analysis.

Several works have appeared testing the validity of the effective mass approximation (EMA) in the past. Either they suggest reformulation or lead to more numerical procedures unsuitable for the interpretation of experimental data [7-9].

In the present work we have considered a donor electron in a QW subjected to an external magnetic field. The donor binding energies are calculated within the EMA by following a variational procedure. We have considered quantum wells (QWs) of the semimagnetic semiconductor system also. The variations of the barrier height with magnetic field are considered using a simple model presented in [10]. The above calculations were repeated using position dependent effective mass (PDEM) as suggested in [11]. Finally the application of the EMT to the QW case is critically examined in section V.

The paper is organized as follows. In section II we present the theoretical framework. PDEM will be presented in section III and a brief discussion of semimagnetic semiconductors will be given in section IV. We give our results and discussion in section V. Main conclusions are summarized in section VI.

\section{THEORETICAL FRAMEWORK}

\section{A. Infinite well model}

The Hamiltonian for a donor impurity in the presence of a magnetic field applied along the z-direction in a square $\mathrm{QW}$ is given by

$$
\mathrm{H}=\left(\frac{1}{2 m^{*}}\right)\left(\mathrm{P}+\frac{e \mathrm{~A}}{c}\right)^{2}-\frac{e^{2}}{\varepsilon r}+V_{W}(z)
$$

where $m *$ is the effective mass of the donor electron, A the 
TABLE I: Material parameters for non -magnetic and magnetic quantum wells ${ }^{+}$

\begin{tabular}{|c|c|c|c|c|}
\hline Parameter & & terial & Symbol & Unit \\
\hline Lattice Constant & $\begin{array}{l}\text { GaAs- } \\
\mathrm{Ga}_{1-\mathrm{x}} \mathrm{Al}_{\mathrm{x}} \mathrm{As} \\
5.69\end{array}$ & $\begin{array}{l}\text { CdTe- } \\
\mathrm{Cd}_{1-\mathrm{x}} \mathrm{Mn}_{\mathrm{x}} \mathrm{Te} \\
\quad 6.48\end{array}$ & $\mathrm{a}$ & $\AA$ \\
\hline $\begin{array}{l}\text { Static Dielectric } \\
\text { Constant }\end{array}$ & 12.65 & 10.9 & $\varepsilon$ & - \\
\hline $\begin{array}{l}\text { Effective Mass } \\
\text { of the Electron }\end{array}$ & $0.067 m_{0}$ & $0.096 m_{0}$ & $m^{*}$ & a.u \\
\hline $\begin{array}{l}\text { Effective Bohr } \\
\text { radius of the } \\
\text { Electron }\end{array}$ & 188.806 & 113.542 & $a^{*}$ & a.u \\
\hline
\end{tabular}

${ }^{+}$Data from Ref. [10] \& [11]

vector potential and $\varepsilon$ the dielectric constant of the well material. The values of the material parameters for non -magnetic and magnetic QWs are given in Table 1.

The confining potential is given by

$$
\begin{aligned}
V_{W}(z) & =0 & |z| \leq \frac{L}{2} \\
& =\infty & |z|>\frac{L}{2}
\end{aligned}
$$

Here $L$ is the size of the QW.

After introducing the effective Rydberg $R^{*}=m^{*} e^{4} / 2 \hbar^{2} \varepsilon^{2}$ as the unit of energy and effective Bohr radius $a^{*}=\hbar^{2} \varepsilon / m^{*} e^{2}$ as the unit of length, the above Hamiltonian takes the new form

$$
\mathrm{H}_{1}=-\nabla^{2}+\gamma L_{z}+\frac{\gamma^{2} \rho^{2}}{4}-\frac{2}{r}+\frac{V_{W}(z)}{R^{*}}
$$

Here $\gamma=\hbar \omega / 2 R^{*}$ is a dimensionless quantity. For GaAs, $\gamma=1$ corresponds to approximately 6 Tesla. The contribution of the second term vanishes for the ground state. We follow the variational procedure to obtain the ground state energy [12]. The ground state wave function of the above Hamiltonian can be written as

$$
\psi_{1}=N_{1} e^{-\alpha \rho^{2}} e^{-\beta z^{2}} \cos \left(\frac{\pi z}{L}\right) \quad|z| \leq \frac{L}{2}
$$

where $\alpha$ and $\beta$ are variational parameters and $N_{1}$ is normalization constant. Yafet et al. used the product Gaussian with two parameters for bulk semiconductors [13]. This form was shown to be accurate for $\gamma>1$ Several subsequent works also employ the form given in Eq. (4) for QW systems with great success [14].

Minimizing $<H_{1 \text { min }}>$ with respect to $\alpha$ and $\beta$, the ionization energies for various magnetic fields can be calculated using the following formula

$$
E_{\mathrm{ion}}=E_{\mathrm{Sub}}+\gamma-<H_{1 \mathrm{~min}}>
$$

$E_{\text {Sub }}$ is the sub band energy in the presence of a magnetic field, which can be calculated when the impurity potential is absent in Eq.(3). Since the zero of energy fixed at the bottom of the well is shifted above by Landau energy $\hbar \omega_{c} / 2$ (which is $\gamma$ expressed in $R^{*}$ ) and the subband energy, $E_{\text {ion }}$ is obtained as in Eq. (5).

\section{B. Finite well model}

The confining potential is given by

$$
\begin{array}{rlr}
V_{W}(z) & =0 & |z| \leq \frac{L}{2} \\
& =V & |z|>\frac{L}{2}
\end{array}
$$

Here $V$ is the barrier height of the QW. GaAs- $\mathrm{Ga}_{1-x} \mathrm{Al}_{x} \mathrm{As}$ being a non-magnetic material, the barrier height doesn't vary with the applied magnetic field. CdTe-Cd ${ }_{1-x} \mathrm{Mn}_{x} \mathrm{Te}$ being a semimagnetic material, the barrier height varies with the applied magnetic field. In any QW of a magnetic material, the barrier height decreases with applied magnetic field for one spin orientation [10].

The ground state wave function of the above Hamiltonian can be written as,

$$
\begin{aligned}
\psi_{2} & =N_{2} e^{-\alpha \rho^{2}} e^{-\beta z^{2}} \cos (\delta z) \quad|z| \leq \frac{L}{2} \\
& =N_{3} e^{-\alpha \rho^{2}} e^{-\beta z^{2}} e^{-\xi|z|} \quad|z| \geq \frac{L}{2}
\end{aligned}
$$

Here

$$
\delta=\sqrt{\frac{2 m^{*} E}{\hbar^{2}}} \quad \text { and } \quad \xi=\sqrt{\frac{2 m^{*}(V-E)}{\hbar^{2}}}
$$

where $\alpha$ and $\beta$ are variational parameters; $N_{2}$ and $N_{3}$ are normalization constants. The ionization energies for various magnetic fields can be calculated using Eq. (5).

\section{POSITION DEPENDENT EFFECTIVE MASSES}

In the infinite barrier model, since tunneling is not allowed, we use

$$
1 / m^{*}(L)=1 / m^{*}+\left(1-1 / m^{*}\right) \exp [-\lambda L]
$$

where $m^{*}$ is the effective mass in the well $\left(0.067 m_{0}\right.$ if the well is GaAs material and $0.096 m_{0}$ if the well is CdTe material). Free electron mass $m_{0}=1$ in a.u and $\lambda$ is a constant which we choose to be $0.01\left(\AA^{-1}\right)$. This choice is chosen keeping in mind the fact that as $L \rightarrow 0$ the particle is strongly 
bound within a $\delta$ - function well, wherein there is no periodicity (in the z-direction), whereas as $L \rightarrow \infty$, the system is 3 dimensional (i.e. bulk) characterized by the conduction band effective mass $m^{*}$. A choice $\lambda^{-1} \leq a^{*}$ looks reasonable.

When the Barrier height is finite, which is always true in all practical situations, in addition to the mass correction given by Eq. (9), another contribution becomes essential. Since the carrier can tunnel through the barrier, the barrier mass should also be considered. We suggest

$$
1 / m^{*}(z)=A\left(1-B z^{2}\right)
$$

where $A$ is as given by the RHS of Eq. (9), $B=\Delta / L^{2}$ and $L$ is the size of the well. $\Delta$ is as given below.

The position dependent effective mass used in Ref [15, 16] is given by $m_{1}^{*}(z)=g(z) m_{1}^{*}=a+b z^{2}$ where $g(z)=$ $1+0.4018 z^{2} / L^{2}$

It is shown that the contribution by PDEM is less than $1 \mathrm{meV}$. We point out two draw backs of this mass variation.

(1) Either in the infinite barrier model or in a realistic situation when $\mathrm{x}$ is large like 0.4 , the probability of finding an electron at the boundary of the well is zero. Hence the above variation does not represent this situation eventhough the authors of $\operatorname{Ref}[15,16]$ use $x=0.32$.

(2) Unlike their claim in Ref [15] that the effective mass tends to the bulk value when $L \rightarrow 0$ and as $L \rightarrow \infty$, we notice that in the limit $L \rightarrow 0$ the effective mass becomes infinity which is un physical. However in this limit for a finite barrier problem, due to tunneling the effective mass should be that of the barrier. To overcome these draw backs we have suggested the mass variation as in Eq. (10).

The derivation of position dependent effective mass $[1 / m(z)]$ of Eq. (10) is as given below. The effective mass of the electron in the well can be written as

$$
1 / m^{*}(z)=a+b z^{2}
$$

Appling the boundary conditions

(i) when $z=0,1 / m^{*}(0)$ becomes ' $a$ ' which is equal to $1 / m_{w}^{*}$ given by Eq. (9).

(ii) when $z=L, 1 / m^{*}(L)$ becomes $1 / m *_{w}+b L^{2}$. We know that the mass at $z=L$ is $1 / m_{b}^{*}$, which is the barrier mass that depends on $x$. The value of $b$ is $1 / L^{2}\left[1 / m_{b}^{*}-1 / m_{W}^{*}\right]$. The effective mass of GaAs in the barrier varies with composition as $m *_{b}(x)=0.067+0.0835 x$ for $0 \leq x \leq 0.4$.

Eq.(11) is modified as

$$
\begin{aligned}
\frac{1}{m^{*}(z)} & =\frac{1}{m_{W}^{*}}-\frac{z^{2}}{L^{2}}\left(\frac{1}{m_{W}^{*}}-\frac{1}{m_{b}^{*}}\right) \\
& =\frac{1}{m_{W}^{*}}\left[1-\frac{z^{2}}{L^{2}}\left(\frac{0.0835 x}{m_{W}^{*}}\right)\right] \\
& =\frac{1}{m_{W}^{*}}\left[1-\frac{\Delta z^{2}}{L^{2}}\right]
\end{aligned}
$$

where $\Delta=0.0835 x / m_{w}^{*} \pi \pi 1$ for GaAs-Ga ${ }_{1-x} \mathrm{Al}_{x} \mathrm{As}$ system.
In the present work we extend our earlier work [11] to the case of CdTe-Cd $\mathrm{Cd}_{1-x} \mathrm{Mn}_{x} \mathrm{Te}$, which is a semimagnetic material. The effective mass of CdTe in the barrier region varies with composition as, $m_{b}^{*}(x)=0.096+0.067 x$ for $0 \leq x \leq 0.7$ for $\mathrm{CdTe}-\mathrm{Cd}_{1-x} \mathrm{Mn}_{x} \mathrm{Te}$ system.

Eq. (12) is valid with $\Delta=0.067 x / m_{w}^{*} \pi \pi 1$ and we have chosen $x=0.2$ for both the systems.

After simplifying the expression for $\Delta$, we obtain $\Delta=0.2$ and 0.123 for GaAs- $\mathrm{Ga}_{1-x} \mathrm{Al}_{x}$ As and CdTe-Cd ${ }_{1-x} \mathrm{Mn}_{x} \mathrm{Te} \mathrm{QW}$ systems respectively. The present results of mass variation are compared with the one used in $\operatorname{Ref}[15,16]$ as given in Fig. 1.

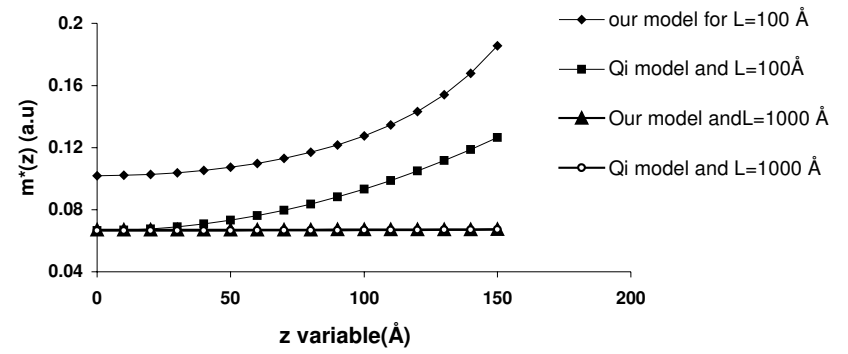

FIG. 1: Variation of effective mass in the well region for two different models.

In the present work, our aim is to check whether the use of a PDEM affects the donor ionization energy or not. The effects of such a PDEM on the donor binding energies in the presence of an external perturbation such as a magnetic field, that is applied along the growth direction of QW, is discussed using perturbation method for infinite and finite barrier confinements.

The perturbation for infinite QW is chosen as

$$
\mathrm{H}^{1}=-\frac{\hbar^{2}}{2}\left(\frac{1}{m^{*}(L)}-\frac{1}{m_{w}^{*}}\right) \nabla^{2}
$$

and for finite QW,

$$
\mathrm{H}^{1}=-\frac{\hbar^{2}}{2}\left(\frac{1}{m^{*}(z)}-\frac{1}{m_{w}^{*}}\right) \nabla^{2} .
$$

We replace

$$
\frac{1}{2}\left(\frac{1}{m^{*}(z)}\right) \nabla^{2}
$$

as

$$
\frac{1}{4}\left[\frac{1}{m^{*}(z)} \nabla^{2}+\nabla^{2} \frac{1}{m^{*}(z)}\right]
$$

The expressions for $\left(1 / m^{*}(L)\right)$ and $1 / m^{*}(z)$ are as given in Eqs. (9) and (10).

The effect of the position dependent effective mass on subband energy and ionization energies are obtained by perturbation method. The first order energy corrections are given by $\Delta E^{(1)}=\left\langle\Psi, \mathrm{H}^{1} \Psi\right\rangle$, where $\Psi$ is the ground state wave function given by Eq.(4) for infinite barrier and Eq.(7) for finite barrier QW. 


\section{SEMIMAGNETIC SEMICONDUCTORS}

Diluted magnetic semiconductors are semiconductor compounds in which some cations are randomly substituted by magnetic ions. The most widely studied alloys are II-VI semiconductors containing manganese, particularly $\mathrm{Cd}_{1-x} \mathrm{Mn}_{x} \mathrm{Te}$. This (wide gap semiconductor) diluted magnetic quantum well system has received immense attention due to the interesting properties arising from the strong exchange interaction between the magnetic ions and the carriers of conduction and valence bands. One of the interesting properties of the semi magnetic semiconductor is the barrier height decrease in a magnetic field. The variation of the band gap difference with magnetic field is given by [17] $\left(\Delta E_{g}^{B} / \Delta E_{g}^{0}\right)=\eta e^{-\xi \gamma}-1 / \eta-1$ where $\Delta E_{g}^{B}$ and $\Delta E_{g}^{0}$ are the band gap differences with and without magnetic field . The band gap of the material is given by [18] $\mathrm{Eg}\left(\mathrm{Cd}_{1-x} \mathrm{Mn}_{x} \mathrm{Te}\right)=1606+1587 x(\mathrm{meV})$. Using the experimentally available critical magnetic fields at which Type I to Type II superlattice transition occurs for acceptors, the results for the donors may be obtained by using the procedure as given in [10]. Fig. 2 shows the variation of barrier height with magnetic field.

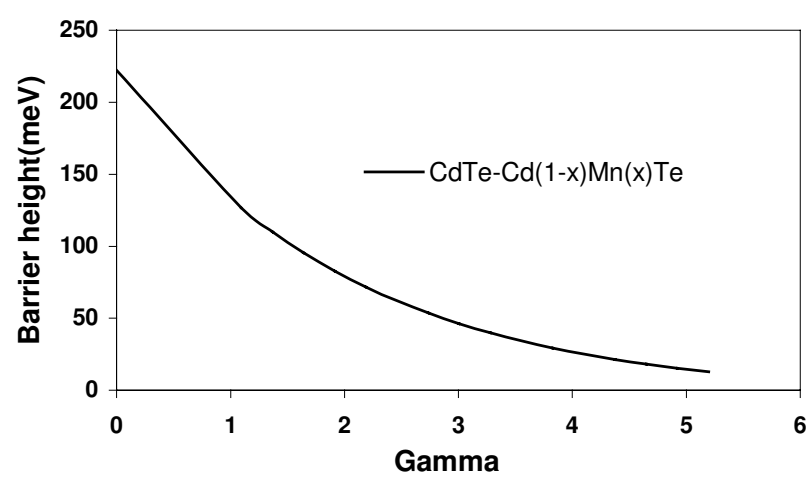

FIG. 2: Variation of barrier height with magnetic field [Ref.10].

\section{RESULTS AND DISCUSSION}

The variation of ionization energy with magnetic field in the infinite well model for non-magnetic QW is shown in Table 2. We conclude that the ionization energy increases with magnetic field for all well dimensions. This result is in agreement with other works $[18,19]$. But at the same time the ionization energy due to PDEM is always greater than the ionization energy due to constant effective mass for all well dimensions $L \leq a^{*}$. We found similar enhancements in an electric field in our earlier work [20]. The ionization energies of Ref [18, 19] are somewhat lower than our results. This is due to the neglect of the magnetic field effect on the subband energies in those references.

The results for the variation of subband energy for a finite barrier magnetic quantum well, in the presence of magnetic field, shows that the subband energy decreases as the well size increases, which is a consequence of quantum size effect. The PDEM drastically reduces the subband energy up to $L=100 \AA$ for all applied magnetic fields. It also follows that the effect of PDEM is unimportant when the well dimension is greater than the effective Bohr radius.

We have also seen how the subband energy varies with well size in the presence of a magnetic field in a finite non -magnetic QW. In general, the PDEM affects the subband energies for narrow wells such that $L \leq a *$. Also the subband energy decreases in the presence of PDEM.

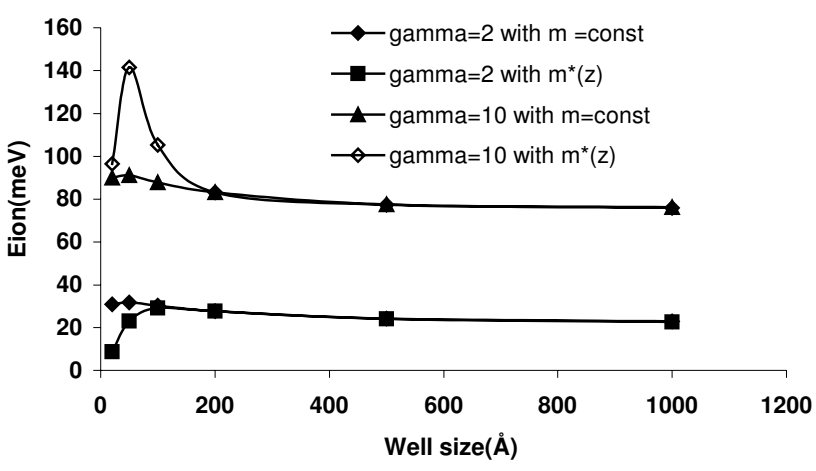

FIG. 3: Variation of ionization energy with well size-finite barrier model.

Figure 3 shows the results of the variation of ionization energy of non -magnetic QW with finite barrier, in the presence of two different magnetic fields. In the case of strong applied magnetic field, we observe that the ionization energy due to $m *(z)$ is greater than the ionization energy due to constant effective mass.

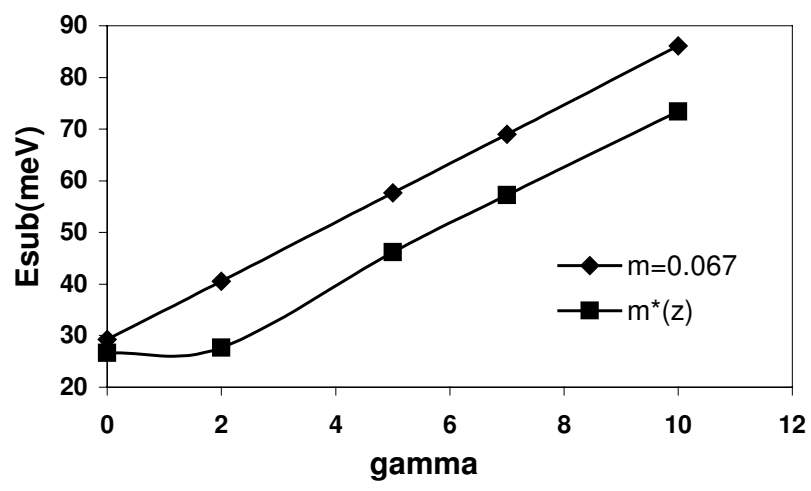

FIG. 4: Variation of subband energy with magnetic field for $\mathrm{L}=100 \AA$.

Figure 4 shows the variation of sub band energy with magnetic field for the finite non - magnetic QW having the dimension of $100 \AA$ for constant effective mass and PDEM. The effect of PDEM on the sub band energy is appreciable.

The variation of ionization energy with well size for the infinite magnetic QW for two different effective masses has also been studied. Here also PDEM affects the energy for narrow wells. We present our results of the variation of ionization en- 
TABLE II: Variation of effective mass in the well region for two different models.

\begin{tabular}{|c|c|c|c|c|c|c|c|c|c|c|}
\hline \multirow[t]{3}{*}{$\gamma$} & \multicolumn{10}{|c|}{$L(A)$} \\
\hline & \multicolumn{2}{|c|}{50} & \multicolumn{2}{|c|}{100} & \multicolumn{2}{|c|}{200} & \multicolumn{2}{|c|}{300} & \multicolumn{2}{|c|}{500} \\
\hline & I & II & I & II & I & II & I & II & I & II \\
\hline 2 & 34.49 & 37.84 & 31.81 & 32.77 & 28.51 & 28.59 & 26.49 & 26.50 & 24.31 & 24.40 \\
\hline 5 & 59.94 & 63.39 & 55.94 & 60.94 & 51.07 & 51.15 & 48.25 & 48.25 & 45.49 & 45.46 \\
\hline 7 & 75.45 & 79.03 & 70.66 & 71.64 & 64.93 & 65.04 & 61.71 & 61.74 & 58.74 & 58.72 \\
\hline 10 & 97.58 & 100.20 & 91.72 & 92.75 & 84.89 & 85.01 & 81.25 & 81.54 & 78.07 & 78.07 \\
\hline
\end{tabular}

\# In all cases I and II refer to $m^{*}=0.067$ a.u and $m^{*}(z)$ respectively.

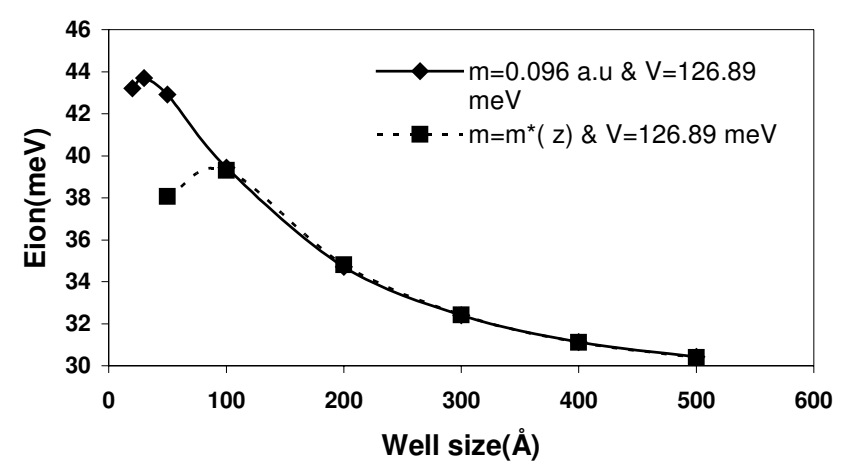

FIG. 5: Variation of ionization energy with well size in the finite barrier model for CdTe-Cd(1-x)Mn(x)Te system. ergy in a finite magnetic QW in the presence of magnetic field for two different masses in Fig. 5. A similar trend is observed.

The variation of sub band energy with well size for the infinite magnetic QW in the absence of magnetic field has also been investigated. The effect of PDEM is low for well dimensions $L \leq a^{*}$.

In the QW case the applicability of EMT requires the following criterion has to be satisfied. Since $E_{\text {ion }} / \Delta E_{1,2}$ should be $<<1$, where $\Delta E_{1,2}$ is the separation between the first two subbands and $a^{*} / L$ should also be less than 1 , we obtain the following criterion: $E_{\text {ion }} a^{*} / \Delta E_{1,2} L<<1$.

To calculate the energy of the first excited sub band, we have chosen a wave function

$$
\begin{aligned}
\psi_{3} & =N_{4} e^{-\alpha \rho^{2}} e^{-\beta z^{2}} \sin \left(\frac{2 \pi z}{L}\right) \quad|z| \leq \frac{L}{2} \quad \text { in the infinite well case, } \\
\psi_{4} & =N_{5} e^{-\alpha \rho^{2}} e^{-\beta z^{2}} \sin (\delta z) \quad|z| \leq \frac{L}{2} \\
& =N_{6} e^{-\alpha \rho^{2}} e^{-\beta z^{2}} e^{-\xi|z|} \quad|z| \geq \frac{L}{2} \quad \text { in the finite well case }
\end{aligned}
$$

and followed the variational method [12] to obtain the energy. These wave functions may be easily seen to be orthogonal to the ground state subband wave functions, Eqs. (4) and (7). The obtained results are summarized in Table 3 and Table 4. In all cases, as can be seen from these tables, the EMT is not violated irrespective of whether one uses the constant effective mass or a PEDM.

The second criterion for the validity of EMT, as given in the introduction, demands $|\kappa| / \kappa_{B Z} \pi \pi 1$. This condition is automatically satisfied for shallow donors in bulk semiconductors, since $a / a^{*} \pi \pi 1$. However, in superlattice systems one may have to consider mini Brillouin zones for which $\kappa_{B Z} \cong \pi / L$, where $L$ is the super lattice period. Hence the criterion $|\kappa| / \kappa_{B Z} \cong L / \pi a^{*} \pi 1$, for GaAs, would mean
$L \pi 300 \AA$. However, in the case of an isolated quantum well, which alone is considered in the present work, this criterion coincides with the above, as in bulk.

\section{CONCLUSIONS}

In this work, we have considered the effects of PDEM and magnetic field in the estimation of donor ionization energies of GaAs- $\mathrm{Ga}_{1-x} \mathrm{Al}_{x}$ As and CdTe-Cd ${ }_{1-x} \mathrm{Mn}_{x}$ Te quantum wells with two different confinements. The PDEM leads to appreciable contributions to the donor ionization energies for narrow wells. Since the contributions are larger, we verify the validity of EMT in all cases studied. We do not find any vio- 
TABLE III: Validity of EMT for an isolated non-magnetic inifinite quantum well in different magnetic fields $\$$.

\begin{tabular}{|c|c|c|c|c|c|c|c|c|c|c|c|c|c|c|}
\hline \multirow[t]{3}{*}{$\begin{array}{l}\text { Well } \\
\text { size }\end{array}$} & \multicolumn{6}{|c|}{$\begin{array}{l}E_{2} \text { sub } \\
(\mathrm{meV})\end{array}$} & \multicolumn{8}{|c|}{$\frac{E_{\text {ion }}(\gamma) a^{*}(\gamma)}{\Delta E_{1,2}(\gamma) L}$} \\
\hline & \multicolumn{2}{|c|}{$\gamma=2$} & \multicolumn{2}{|c|}{$\gamma=5$} & \multicolumn{2}{|c|}{$\gamma=10$} & \multicolumn{2}{|c|}{$\gamma=0$} & \multicolumn{2}{|c|}{$\gamma=2$} & \multicolumn{2}{|c|}{$\gamma=5$} & \multicolumn{2}{|c|}{$\gamma=10$} \\
\hline & I & II & I & II & I & II & I & II & I & II & I & II & I & II \\
\hline 50 & 908.65 & 579.54 & 925.72 & 593.51 & 954.17 & 616.79 & 0.017 & 0.026 & 0.017 & 0.027 & 0.017 & 0.027 & 0.017 & 0.027 \\
\hline 100 & 235.70 & 203.06 & 252.77 & 218.92 & 281.22 & 245.36 & 0.034 & 0.039 & 0.034 & 0.039 & 0.034 & 0.039 & 0.034 & 0.039 \\
\hline 200 & 67.46 & 66.13 & 84.53 & 83.02 & 112.98 & 111.17 & 0.068 & 0.069 & 0.068 & 0.069 & 0.068 & 0.069 & 0.068 & 0.069 \\
\hline 500 & 20.35 & 20.35 & 37.42 & 37.42 & 65.87 & 65.87 & 0.169 & 0.169 & 0.169 & 0.169 & 0.169 & 0.169 & 0.169 & 0.169 \\
\hline$\$$ & U & o & 1) & & $\mathrm{E}_{\text {ion }}$ & & & & & & & & & \\
\hline
\end{tabular}

TABLE IV: Validity of EMT for an isolated non-magnetic finite quantum well in different magnetic fields*.

\begin{tabular}{|c|c|c|c|c|c|c|c|c|c|c|c|c|}
\hline $\begin{array}{l}\text { Well } \\
\text { size }\end{array}$ & \multicolumn{6}{|c|}{$\begin{array}{l}E_{2} \text { sub } \\
(\mathrm{meV})\end{array}$} & \multicolumn{6}{|c|}{$\frac{E_{\text {ion }}(\gamma) a^{*}(\gamma)}{\Delta E_{1,2}(\gamma) L}$} \\
\hline & \multicolumn{2}{|c|}{$\gamma=0$} & \multicolumn{2}{|c|}{$\gamma=2$} & \multicolumn{2}{|c|}{$\gamma=5$} & \multicolumn{2}{|c|}{$\gamma=0$} & \multicolumn{2}{|c|}{$\gamma=2$} & \multicolumn{2}{|c|}{$\gamma=5$} \\
\hline$(\AA)$ & I & II & I & II & I & II & I & II & I & II & I & II \\
\hline 100 & 108.54 & 99.05 & 119.67 & 94.01 & 136.74 & 115.92 & 0.072 & 0.079 & 0.072 & 0.086 & 0.072 & 0.082 \\
\hline 200 & 39.32 & 38.24 & 50.58 & 46.52 & 67.65 & 64.03 & 0.097 & 0.101 & 0.097 & 0.104 & 0.097 & 0.102 \\
\hline 500 & 7.78 & 7.68 & 19.14 & 18.66 & 36.21 & 35.55 & 0.195 & 0.198 & 0.196 & 0.202 & 0.196 & 0.206 \\
\hline
\end{tabular}

lation of EMT in isolated QW cases. The effect of PDEM on the critical concentrations of donors for Metal-Insulator Transition (MIT) in QW systems will form the basis for another publication.
We hope that the present work will be useful in interpreting all the experimental data relevant to optical and electronic properties of doped QW systems as the EMT is vigorously tested and found to be valid.
[1] W. Kohn, Solid State Phys. 5, 257 (1957).

[2] R. Resta, In Crystalline Semiconducting Materials and Devices, edited by P. N. Butcher, N. H. March, and M. P. Tosi (Plenum Press) 217, 1986.

[3] M. Altarelli, W. Y. Hsu, Phys. Rev. Lett. 43, 1346 (1979).

[4] G. Bastard, Phys. Rev. B 24, 4714 (1981).

[5] K. Goser, P. Golsekotter, and J. Dienstubl, Nano electronics and Nano systems (Springer-verlag, Newyork) 2004

[6] R. K. Willardson, A. C. Beer, and E. R. Weber Ed, Nano Structural systems-Semiconductors and Semimetals 35, (1992)

[7] V. Mlinar, M. Tadic, B. Partoens, and F. M. Peeters, Phys. Rev. B 71, 205305 (2005).

[8] C. Priester, G. Allan, and M. Lannoo, Phys. Rev. B 28, 7194 (1983).

[9] G. T. Einevoll, Phys. Rev. B 42, 3497 (1990).
[10] A. John Peter, K. Gnanasekar, and K. Navaneethakrishnan, Eur. Phys. J. B 53, 283 (2006).

[11] S. Rajashabala, K. Navaneethakrishnan, Mod. Phys. Lett. B 20, 1529 (2006).

[12] L. I. Schiff, Quantum Mechanics 3rd Edition (McGraw Hill, Singapore) 1968.

[13] Y. Yafet, R. Keyes, and E. N. Adams, J. Phys. Chem. Solids 1, 137 (1956).

[14] A. Elangovan, K. Navaneethakrishnan, J. Phys. Condensed Matter 5, 4021 (1993).

[15] Yu-Xian-Li, Jian-Jun Liu, and Xiao-Jun Kong, J. App. Phys.88, 2588 (2000).

[16] X.-H.Qi, X.-J.Kong, J.-J.Liu, Phys. Rev. B 58, 10578 (1998).

[17] S. G. Jayam, K. Navaneethakrishnan, Int. J. Mod. Phys. B 16, 3737 (2002). 
[18] F. Long, P. Harrison, and W. E. Hagston, J. Appl. Phys.79, 6939 (1996).

[19] R. L. Greene, K. K. Bajaj, Phys. Rev.B 31, 913 (1985).
[20] S. Rajashabala, K. Navaneethakrishnan, Mod. Phys. Lett. B (communicated). 